\title{
Aprendizaje de las fracciones en estudiantes de séptimo grado del Instituto San Ramón, en Río San Juan de Nicaragua
}

\author{
Paulino Alemán Gudiel ${ }^{1}$ \\ José Esteban Alemán Gudiel ${ }^{2}$ \\ Eugenio Casimiro López Mairena ${ }^{3}$
}

\section{Resumen}

Esta investigación ha analizado el aprendizaje de las fracciones en estudiantes de séptimo grado del Instituto San Ramón, en Río San Juan de Nicaragua. Fue de carácter cualitativa con la participación de 12 discentes de séptimo grado y un docente de matemática. Se aplicaron instrumentos como el test cognitivo, entrevista y la observación.

Los resultados muestran que el estudiantado tiene dificultes de aprendizajes por falta de conocimientos previos, falta de interés y se evidencia inasistencia a las clases de matemáticas. Además, se evidencia que las competencias con el abordaje de la temática son empíricas. Todo lo cual conlleva a la incomprensión de las matemáticas. Es por ello, que se ha propuesto un plan que contribuya al mejoramiento del conocimiento didáctico y matemático de las fracciones de séptimo grado de Educación Secundaria.

Palabras clave: aprendizaje; fracciones; dificultades de aprendizaje; competencias.

\section{Summary}

This research has analyzed the learning of fractions in seventh grade students of San Ramón Institute, Río San Juan of Nicaragua. It was a qualitative research with the participation of 12 students of seventh grade and the mathematics teacher. Instruments such as cognitive test, interview and observation were applied.

The results show that the students have difficulty in learning due to lack of prior knowledge, lack of interest and lack of attendance to mathematics classes. In addition, it is evident that the competences with the approach of the subject are empirical. All of which leads to misunderstanding of mathematics. It is for this reason that a plan has been proposed that contributes to the improvement of the didactic and mathematical knowledge of fractions in seventh grade of Secondary Education.

1 Licenciado en Ciencias de la Educación con Mención en Matemática. Profesor de Matemáticas del Ministerio de Educación de Río San Juan. Correo: paulinoalemangudiel616@gmail.com

2 Licenciado en Ciencias de la Educación con Mención en Matemática. Profesor de Matemáticas del Ministerio de Educación de Río San Juan. Correo: alemanesteban91@gmail.com

3 Doctor en Innovación en la formación. Vicerrector de la Universidad de las Regiones Autónomas de la Costa Caribe Nicaragüense. Correo: eugenio.lópez@uraccan.edu.ni 
Key Words: Learning, fractions, learning difficulties, competences.

\section{Introducción}

Esta investigación analiza el proceso de aprendizaje de las fracciones en estudiantes de séptimo grado del instituto San Ramón en Río San Juan de Nicaragua. Existe una problemática de aprendizaje de las fracciones, por ejemplo, dificultades en la interpretación de las definiciones de conceptos, es decir, deficiencia en la interpretación a lo que indica, significa y representa una fracción. También, dificultades en el uso de algoritmos o procesos para realizar las operaciones con fracciones. Cabe mencionar que, estas dificultades son consecuencias de la incidencia de situaciones que están dentro del aprendizaje de las fracciones.

En este sentido, se estudian las dificultades de aprendizaje en las fracciones a través de aplicación y análisis de instrumentos como la observación, cuestionario cognitivo y entrevista al docente de matemáticas. Esto permitirá, identificar factores que inciden en el aprendizaje de las fracciones tales como: conocimientos previos, inasistencias, la metodología de enseñanza aprendizaje, entre otros. Por ello, se analizan las metodologías que el docente usa en el aula de clases; los conocimiento adquiridos por el estudiantado sobre las fracciones y la perspectiva del profesorado acerca de este aprendizaje.

\section{Revisión de literatura}

Se define que el aprendizaje es el proceso a través del cual se adquieren o modifican habilidades, destrezas, conocimientos, conductas o valores como resultado del estudio, la experiencia, la instrucción, el razonamiento y la observación (Pulido, 2007). Este proceso puede ser analizado desde distintas perspectivas por lo que existen diferentes teorías del aprendizaje. Dickson, Brown y Gibson (1991), agregan que las fracciones, son los números racionales expresables como razón o cociente de dos números enteros y, Baltodano (2015) define el concepto de fracción como una parte de un número entero; expresa las partes iguales en las que se divide un todo que llamamos unidad y cuantas de esas partes se toma. En definitiva, una fracción es una porción de un todo, además como quebrado, número que expresa una o varias partes de la unidad dividida en partes iguales.

Es por ello, que el concepto de fracción puede limitarse a cierto contraste matemático, y el abordaje según la relación "parte-todo" es limitado fenomenológicamente y también matemáticamente (Freudenthal, 1994). Desde esta perspectiva, Vergnaud (1998) afirma que el concepto de fracción comprende dos relaciones fundamentales: La relación parte-todo y la parte-parte. El autor resalta algunas características básicas para la adquisición de ese contenido matemático, en que se debe comprender que una fracción implica un determinado número de partes. Ante esto se puede validar que 
las fracciones están detrás de muchas de las actividades que se realizan en la vida del ser humano.

En relación con la problemática en el aprendizaje de las fracciones se han encontrado dificultades que influyen en la formación de estudiantes del séptimo grado de educación secundaria relacionadas con el reconocimiento de los conceptos, propiedades y procedimientos de resolución de fracciones (Pego (2012). Así mismo, Vergnaud (1998) argumenta que existe un interés didáctico sobre el aprendizaje de las fracciones puesto que son instrumentos para el análisis de las dificultades conceptuales, obstáculos cognitivos, procedimientos y las posibles representaciones que configuran el estudiantado.

También, Vásquez (2012) afirma que el estudiantado presenta dificultad de aprendizaje cuando enfrentan o intentan trabajar con situaciones que están basadas en fracciones, funciones racionales y ecuaciones con coeficientes fraccionarios. Los conceptos y teoremas son importantes porque en la primera entrada de un campo conceptual son las situaciones-problemas y la segunda los conceptos y teoremas (Vergnaud, 1990). Ante esto la comprensión del concepto de fracción es acorde en cómo se entienda cada significado. Respecto a la comprensión Llinares y Sánchez (1997) mencionan que llegar a la comprensión del concepto de fracción es un largo camino debido a sus múltiples interpretaciones, sin mencionar a las ya establecidas desde el lenguaje cotidiano, cuestión que suele estar presente en los procesos de aprendizaje de estos temas (p. 189). Los obstáculos son situaciones importantes que inciden en el aprendizaje de fracciones. De acuerdo con Arbeláez (2015), un obstáculo es algo que impide avanzar en el aprendizaje de algún concepto, en la oportunidad para avanzar, en un conocimiento deficiente que es transmitido por el docente, por la dificultad que manifiesta para transmitirlo. De acuerdo a lo anterior, el estudio de las fracciones es un momento importante en el aprendizaje de las matemáticas (Achondo, 2010). Siguiendo a este autor, actualmente se registran dificultades en el aprendizaje debido a las diversas representaciones conceptuales que admite este concepto. Además, el dominio de las fracciones, es un campo conceptual constituido por un conjunto de situaciones cuyo dominio progresivo requiere de una variedad de procedimientos, de conceptos y representaciones simbólicas que están en estrecha conexión.

\section{Materiales y métodos}

Esta investigación se sustenta en la metodología cualitativa porque es una actividad sistemática orientada a la comprensión en profundidad de fenómenos educativos y sociales, a la transformación de prácticas y escenarios socioeducativos, a la toma de decisiones y al descubrimiento y desarrollo de un cuerpo organizado de conocimiento (Bisquerra, 2012). A partir de las aportaciones de estos expertos, nuestra investigación se centró en el análisis del proceso de aprendizaje de las fracciones en estudiantes de séptimo grado del Instituto San Ramón de Río San Juan de Nicaragua. El abordaje 
sigue el diseño fenomenológico, porque se trata de describir las experiencias individuales subjetivas del grupo de participantes (Hernández et al., 2010).

En este estudio la principal fuente de información son el estudiantado de séptimo grado y la docente del Instituto de San Ramón, en Río San Juan de Nicaragua. Para recolectar la información sobre los factores asociados al aprendizaje de las fracciones se aplicaron instrumentos como test cognitivos, entrevistas y guías de observación. Para la aplicación de estos instrumentos se tomó en consideración el consentimiento previo libre e informado de los participantes, y la autorización de las autoridades del Centro Escolar. Cabe mencionar que en estricto rigor ético, los instrumentos tenían un carácter anónimo y fue complementado por los sujetos participantes en presencia del profesorado.

\section{Resultados y discusión}

El estudio evidencia que el estudiantado tiene dificultad de aprendizaje de las fracciones, debido a que no tienen el mismo ritmo al momento de interpretar, analizar y comprender los objetos matemáticos relacionados a esta temática de estudio. Este resultado es congruente con la postura que tiene Vásquez (2012), al señalar que al estudiantado se le hace difícil la compresión de las fracciones. Se suma a ello, la falta de disposición e interés por algunos de los educandos conllevando a una baja competencia en el nivel educativo.

Es importante mencionar que, el estudiantado no se identifica con la disciplina y menos con lo relacionado a fracciones, algunos comentan que dicha temática no la reciben en la formación básica, ante esto se debe de ser pertinente para que en la educación media se facilite el aprendizaje de los niños y niñas; a esto Achondo (2010) hace referencia de las situaciones implicadas en el aprendizaje, cuyo dominio de estas debe de ser de manera progresiva y coherente en todo el desarrollo del aprendizaje. Con la figura 1, se refleja la importancia que tienen las fracciones.

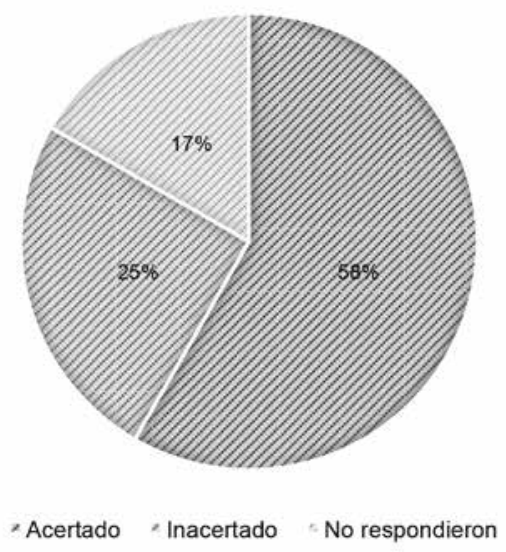

Figura 1: Importancia de las fracciones. 
En este particular se conoce que la mayoría del estudiantado brindaron respuestas que tienen relación con la importancia de las fracciones en la cotidianidad, si bien es cierto se utilizan fracciones para dividir cantidades en las que con los números naturales y en enteros se hace complicado realizar. Relacionado al análisis anterior, Flores y López (2016) señalan que un aspecto importante en el aprendizaje de las matemáticas es tomar en cuenta los contextos personal-familiar, social-comunitario, profesional-especializado y científico-tecnológico donde emergen actividades de la vida cotidiana en el que participa el estudiantado, por ejemplo: medio litro de agua, tres cuartos de tortilla, la ropa está a mitad de precio, y otros.

De acuerdo a Pulido (2007), se presentan ocasiones en las que hacen prácticas de estas situaciones u operaciones con fracciones sin saber la utilización científica. Estas personas lo realizan de manera empírica dentro del mundo de situaciones cotidianas que viven día a día, lo importante es que sus cálculos son aproximados y en ocasiones exactos. En la figura 2, se refleja el conocimiento del estudiantado sobre el concepto de fracción.

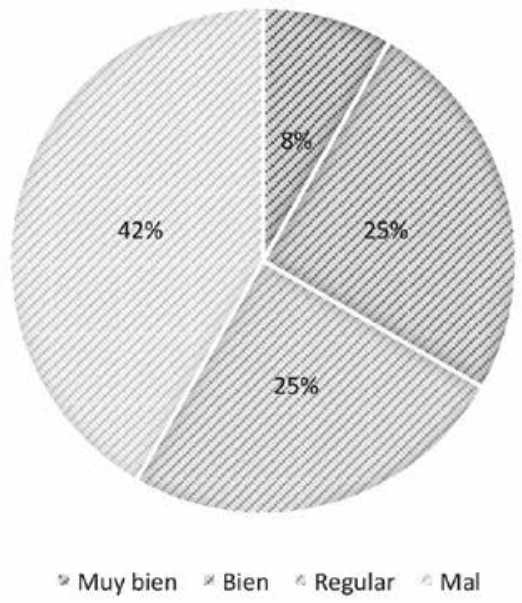

Figura 2: Conocimiento sobre el concepto de fracción.

En la figura 1, observamos que la minoría de estudiantes tiene la noción sobre el término de fracción, por lo que se considera que no tienen bien clara la definición de una fracción, a estos estudiantes hace falta potencializar esta situación para que tengan un aprendizaje significativo y así no presenten consecuencia de no aprendizaje a causa de esto. El estudiantado escribió ideas relacionadas a la conceptuación de fracción, como la siguiente: "una fracción poseen numerador y denominador", a como lo menciona Jarquín y Díaz (2015) "una fracción es el cociente entre dos números enteros" a y b como lo aborda.

En la resolución de las situaciones prácticas del test cognitivo la clasificación de las fracciones por el estudiantado refleja que se puede hacer esta categorización 
interpretando el concepto y la definición de las diversas fracciones. Por otra parte, se han encontrado dificultades de aprendizaje en referencia con la simplificación de fracciones, es decir, el estudiantado presentó dificultades al desarrollar proceso de dicha operación, esta dificultad es por falta del dominio del procedimiento del algoritmo de simplificación, además, no aplicaron la misma divisibilidad para numerador y denominador. Desde esta perspectivas, los discentes no tienen conocimiento previos para fundamentar el proceso de resolución adecuado, por ello, se debe retroalimentar el aprendizaje del proceso de simplificación de fracciones, en términos de la definición de máximo común divisor de numerador y denominador, en concreto, números divisibles de dichos términos (Martínez y Zamora, 2015).

La figura 3, muestra los resultados de las operaciones de adición de fracciones por parte del estudiantado:

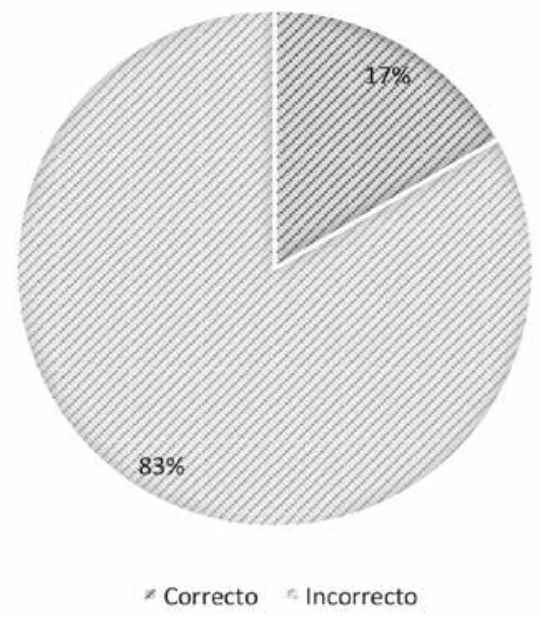

Figura 3: Suma de fracciones.

En el test cognitivo se presentó una suma de fracciones homogéneas y solamente 2 de los 12 estudiantes realizaron bien la operación de adición de fracciones, que equivale al $17 \%$ de los participantes que se incluyó para el estudio, por tanto la mayoría desarrollo mal el proceso que se requería para dar la solución pertinente, lo cual esto representa a un $83 \%$ de dichos participantes. Esto afirma que la mayoría del estudiantado tienen gran dificultad para sumar fracciones.

La figura 4, refleja los resultados de resta de fracciones por parte del estudiantado que participó en el estudio. 


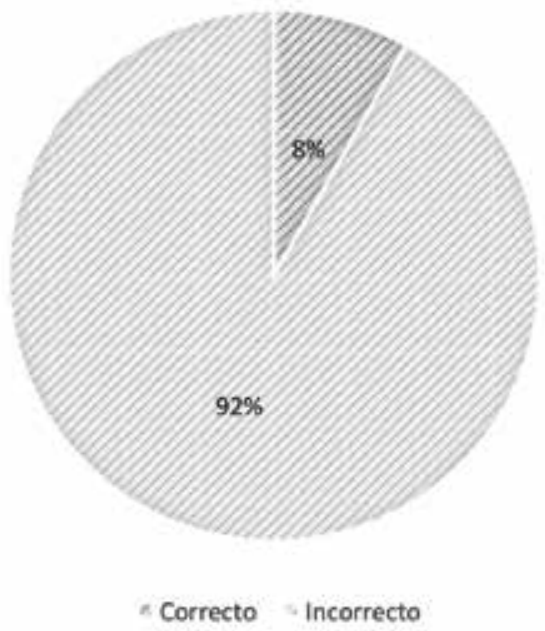

Figura 4: Sustracción de fracciones.

Se presentó una sustracción de fracciones homogéneas y solamente 1 de 12 estudiantes realizó adecuadamente el proceso de resta, lo anterior corresponde a un $8 \%$ de los participantes, por lo tanto un $92 \%$ de los estudiantes desarrollaron una solución errónea. En este apartado de adicción y sustracción de fracción se identificó que la mayoría del estudiantado tienen dificultad para realizar el proceso correcto para efectuar las operaciones indicadas, esto significa que no dominan el algoritmo de suma y resta de fracciones, ya que la mayoría en el desarrollo de las situaciones-problemas relacionados con la adición de fracciones sumaban los denominadores e igual realizaron en las soluciones referidas a los ejercicios con la sustracción de fracciones, es decir, restaban los denominadores. Desde esta perspectiva, se considera que es importante que los educandos tengan un aprendizaje eficiente y potencial para el desarrollo y proceso adecuado para efectuar dichas operaciones con igual denominador (Orozco, 2015).

Se consultó a la docente sobre su práctica didáctica y pedagógica en el aprendizaje de las fracciones, la docente expresa que las fracciones son muy importantes ya que muestra al estudiante maneras de resolver problemas que se vivencian a diario en el contexto real y cotidiano. Así mismo, la docente afirma que el concepto de fracción le permite ir construyendo formas o maneras de realizar y resolver situaciones prácticas relacionadas con el día a día del estudiante, además, que el estudiantado se conocen y descubren que no solo existen números enteros, sino que también fraccionarios. Como estudiantes esta importancia la valoramos como necesaria para la solución de situaciones reales.

Aunque, se encontró que la implementación de estrategias metodológicas por la docente, no se relacionan con la práctica didáctica en el aula, esto puede ser por desconocimiento y disposición de la docente. Según Flores y Salinas (2013) estos contenidos de fracciones conllevan a implementar metodologías propias congruentes con 


\section{EDUCACIÓN}

el contexto social en el que convive el estudiantado. Las prácticas de metodologías permiten un ambiente de aprendizaje para el estudiantado más interesante, en la mayoría de las aulas donde se dan estos contenidos. La docente relató que existen factores que limitan el aprendizaje como: falta de práctica de conocimientos previos, la insuficiencia de las horas clases para desarrollar y ampliar todos los contenidos, y el desinterés e inasistencia del estudiantado.

La docente expresó que los conocimientos previos son importantes ya que desde la práctica didáctica y pedagógica se vivencian en todos los contenidos matemáticos, especialmente relacionados con las fracciones, además, que las dificultades de aprendizaje son más relevantes por la ausencia de dichos conocimientos. En este sentido, porque permitirían mejorar y fortalecer el aprendizaje instruyéndolo de manera más fácil y pertinente, acorde a la diversidad del estudiantado.

\section{Conclusiones}

En esta investigación se ha analizado el proceso de aprendizaje de las fracciones en estudiantes de séptimo grado del instituto San Ramón de Río San Juan de Nicaragua. El análisis permite plantear que la mayoría del estudiantado no dominan e interpretan la definición del concepto de fracción, tampoco comprenden la importancia y utilidad de las fracciones en la vida o contexto social donde conviven. Es importante mencionar que el estudiantado no ha sido capaz de identificar el tipo de fracción para su respectiva clasificación. Es por ello, que la mayoría presentan dificultades en el proceso y desarrollo del algoritmo para la realización de las operaciones de simplificación, adición y sustracción.

Los factores más relevantes e involucrados que se presentaron en la incidencia del aprendizaje fueron: la inasistencia de algunas y algunos estudiantes, y el hecho de que la docente no tenga como profesión las matemática. Además, el estudiantado, no tiene competencias matemáticas para resolver fracciones que son desarrolladas en la educación primaria, esto se visualiza en las dificultades del estudiantes en sus experiencias previas sobre el aprendizaje de las fracciones, sabiendo que los conocimientos previos son necesarios para desarrollar estos contenidos. Desde esta perspectiva, es importante relacionar muchos ejemplos vinculados a la vida diaria, o sea, contextualizar el proceso de aprendizaje, como una herramienta de comprensión de las fracciones. Asimismo, destinar más tiempo al abordaje de la asignatura y la unidad de los racionales (Achondo, 2010).

Finalmente, decir que se hace imprescindible implementar acciones pedagógicas y didácticas para lograr un aprendizaje de las fracciones a partir de la motivación en el aprendizaje, atención individualizada, retroalimentación de los contenidos, fortalecimiento de las competencias matemáticas del profesorado de educación primaria y secundaria a través de un plan de acción de desarrollo del aprendizaje de la fracciones 
en aspectos conceptuales, procedimentales, actitudinales, pedagógicos y didácticos. Así mismo, profundizar y construir conocimientos competentes.

\section{Lista de referencias}

Achondo, B. (2010). Dificultades u obstáculos en la enseñanza-aprendizaje de las fracciones en educación básica.

Arbeláez, N. M. (2015). Dificultades en la enseñanza de las operaciones con números racionales en la educación secundaria. Bogotá: Universidad Autónoma de Colombia.

Baltodano, B. (2015). Matemática de Séptimo Grado de Educación Secundaria. Managua, MINED.

Bisquerra, R. (2012). Metodología de la Investigación Educativa. Madrid, La Muralla.

Dickson, L., Brown, M., \& Gibson, O. (1991). El aprendizaje de las matemáticas. Madrid, MEC: Labor.

Flores, W., O., \& López, E. (2016). Recursos didácticos y tecnológicos para la enseñanza de la integral definida en el modelo de Universidad Comunitaria Intercultural. Ciencia e Interculturalidad, 18(1), 63-78. DOI: http://dx.doi.org/10.5377/rci. v18i1.3050

Freudenthal, H. (1994). Fenomenología didáctica de las estructuras matemáticas. Textos seleccionados. (L. Puig, Introducción, traducción y notas.) México: CINVESTAV.

Hernández, R, Fernández, C., Baptista, P., (2010). Metodología de la Investigación. México, McGraw-Hill.

Jarquín, H., \& Díaz, F. (2015). Programa de Estudio de Educación Secundaria a Distancia en el Campo. Managua, MINED.

LLinares, S., \& Sánchez, M. V. (1997). Aprender a enseñar, modos de representación y número racional. Madrid, Editorial Síntesis.

Pego, V. P. (2012). Las fracciones: ¿problema de aprendizaje o problemas de la enseñanza. Pilquen-Sección Psicopedagogía, (8), 6.

Pulido, C. (2007). Pensamiento histórico y aprendizaje conceptual. Granada, Universidad de Granada.

Vásquez, G. (2012). Seminario de formación docente como estrategia para mejorar la enseñanza y aprendizaje del concepto de fracción. Medellín: Institución Educativa Yermo y Parres.

Vergnaud, G. (1990). Théorie des champs conceptuels. Recherches en didactique des mathématiques, 10, 47-56.

Vergnaud, G. (1998). A comprehensive theory of representation for mathematics education. The Journal of Mathematical Behavior, 17(2), 167-181. 